\title{
Paved ways through delivery barriers
}

\author{
When designing translationally relevant delivery strategies to overcome the physicochemical and biological \\ barriers to getting therapeutics into the right tissues and cells, building on tried-and-tested concepts often pays off.
}

T he delivery of therapeutics to only the intended tissues or cells is a problem that is hard to tackle, even though there have been myriad advances in biology, materials and medicine, beginning from when tablets were first coated with polymers to protect labile drugs from the highly acidic environment of the stomach. A primary reason for this is that the biology underlying the molecular-, cellular- and tissue-level barriers that obstruct the delivery process is complex, with much of it remaining to be uncovered. Hence, to bypass the limitations of the current oversimplified view of the biology of drug delivery, it might be best to improve on the concepts and approaches that are known to work. This strategy might be most relevant in a translational context, because the 'delivery problem' underpins many of the design and safety considerations related to the formulation, synthesis, preclinical and clinical testing, regulatory approval and commercialization of smallmolecule drugs, biologics, nanoparticle constructs and cellular products. Many of these products fail in clinical trials because even if they reach their target, the secondary effects arising from insufficient targeting or selectivity levels severely limit the dose at which they can be delivered, and thus their effectiveness.

Take encapsulation, slow degradation and PEGylation (that is, the attachment of poly(ethylene glycol) to a therapeutic); these are working strategies used to 'protect' many commercial therapeutics from premature degradation and from clearance by the reticuloendothelial system. The chemotherapeutics liposomal doxorubicin (commercially known as Doxil, for the treatment of many cancers, and the first nanomedicine approved by the US Food and Drug Administration) and carmustine wafers (Gliadel; for the treatment of malignant gliomas; the polymer wafers degrade slowly, releasing the drug) are two well-known examples of the success of these strategies. In this issue, Zhiqiang Cao and colleagues report a simple way of improving encapsulation, specifically the bioavailability of hydrophobic drugs (such as doxorubicin) encapsulated in micelles. They show that surfactants made by covalently linking two chains with highly opposing polarity (a superhydrophobic lipid and a superhydrophilic polymer)
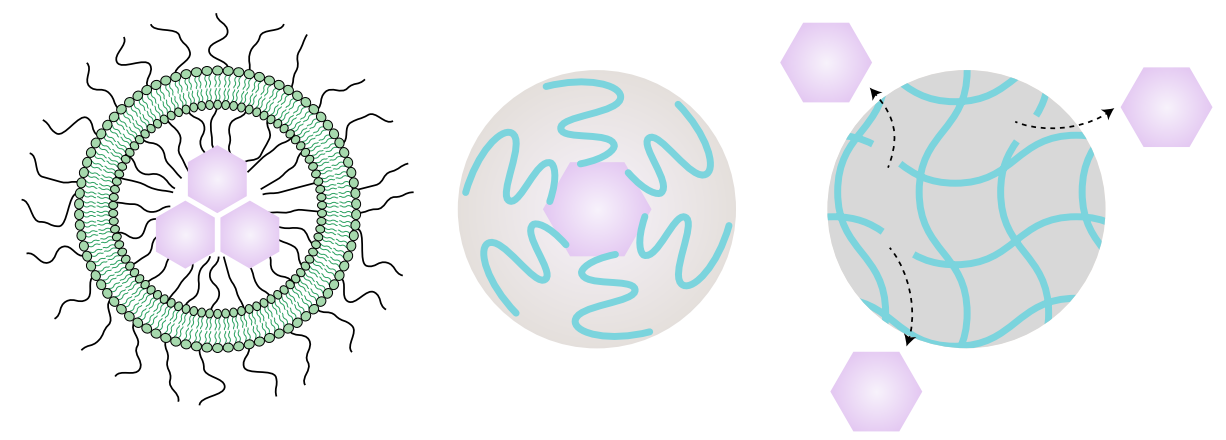

self-organize into micelles that remain ultrastable at highly dilute conditions (in fact, the researchers could not even detect, with currently available methods, the extremely low concentration at which the micelles disassemble). When encapsulating the chemotherapeutic docetaxel, the ultrastable formulation led to the eradication of melanoma tumours in mice under conditions where PEGylated conventional micellar formulations could not reverse tumour growth.

Protection is of course only part of the story. Targeting the right cells selectively requires strategies that often involve overcoming biological and physicochemical barriers. In solid tumours, a number of such barriers are collectively known as the enhanced permeability and retention effect - essentially, a size-based filter enabled by the leaky vasculature and ineffective lymphatic drainage of most tumours, and the mechanism by which most cancer nanomedicines (including the ultrastable micelles of Cao and colleagues) are delivered. But such 'passive' targeting of tumours, although working reasonably well in animal models, has not broadly translated to patients, mostly because of tumour heterogeneity (notably, the degree of vascular leakiness is patient-specific and cancer-type-specific). The targeting of surface receptors overly or specifically expressed on the surface of the targeted cells - the so-called active-targeting approach - offers a higher degree of control. And for drugs that need to be delivered intracellularly, there are additional biological barriers to overcome. For molecules and nanoparticles that are taken up via endocytosis, the processes of escape from endosomal compartments are particularly problematic, in part, because endosomal escape is difficult to observe in vivo.

Xiaohu Gao and colleagues leveraged well-known concepts in protein engineering and self-assembly as well as molecular 'protection' through steric masking to rationally design a selfassembled nanoparticle that combines endosomolytic properties, active targeting, drug encapsulation and PEGylation to more effectively get a synthetic RNA drug that silences a cancer gene into prostate cancer cells, delaying tumour growth in mice. Because of contradictory design requirements for active targeting and the avoidance of toxicity (it is best to avoid positive charge) and for endosomal escape and the encapsulation of negatively charged cargo such as RNA (positive charge is needed), this is a rare case of balancing complexity in functionality with simplicity in design for improved therapeutic efficacy without hampering translatability.

Another rare example of combining known concepts to improve delivery is the marrying of active targeting and protein modification (as in PEGylation) to increase the half-life of therapeutic molecules in circulation, described by Haeshin Lee, Ki-Suk Kim and colleagues. By simply modifying therapeutic proteins and peptides with a natural polyphenol found in plants that specifically accumulates in myocardium, the researchers demonstrate functional recovery of the heart of rats treated after a reperfusion insult (hearttissue damage caused after the return of blood flow following ischaemic injury).

After myocardial infarction, it is crucial that therapeutics are delivered to heart muscle continuously for weeks. Direct injection into the heart is unsurprisingly more effective 
(yet much more invasive) than intravenous delivery. Yet, to achieve extended delivery, Gordana Vunjak-Novakovic and colleagues used an implantable collagen-based hydrogel patch encapsulating and slowly releasing cardiomyocyte-derived extracellular vesicles. Local delivery via the implant led to reduced infarct size and functional recovery of the heart four weeks post-infarction in rats.

Tried-and-tested encapsulation methods are also being applied to immunotherapies. In atherosclerosis, the increase in inflammation of the plaque is associated with macrophages that infiltrate and accumulate in the plaque, aided by activated $\mathrm{T}$ cells. As shown by Willem Mulder, Raphaël Duivenvoorden and colleagues, blocking this interaction can be achieved effectively through a small-molecule inhibitor protected by high-density lipoprotein, as such nanoparticles are taken up by macrophages and inflammatory monocytes to a higher degree than by other cells in the plaque. The researchers show that the approach led to significant reduction of plaque inflammation in mice and that the formulation has low toxicity in both mice and monkeys.

Naturally, when efficacy and translatability are largely irrelevant, as when studying disease mechanisms and refining the biology of delivery barriers, one may need to venture down new roads rather than to rely on proven strategies. Yet on the path to the clinic, improving on paved roads might lead to more efficient delivery.

Published online: 10 May 2018

https://doi.org/10.1038/s41551-018-0245-7 